epilepsy was constantly favorable in $51 \%$, steadily poor in $17 \%$, improving in $25 \%$ and deteriorating in $6 \%$. The number with intractable seizures had not decreased when the 2 year and 5 year outcomes were compared. A model with determinants identified at intake could predict the outcome at 5 years correctly in $64 \%$ of the cohort, especially for a long terminal remission. Prediction was less reliable for a short terminal remission and intractable outcome. (Arts WFM, Brouwer OF, Peters ACB, et al. Course and prognosis of childhood epilepsy: 5-year follow-up of the Dutch study of epilepsy in childhood. Brain August 2004;127:1774-1784). (Respond: Willem F Arts MD, PhD, Department of Paediatric Neurology, Erasmus MC/Sophia Children's Hospital, PO Box 2060, 3000 CB Rotterdam, The Netherlands).

COMMENT. Children with newly diagnosed epilepsy have a favorable prognosis in $76 \%$ of cases and a deteriorating or poor prognosis in $23 \%$. Of variables predicting a poor outcome, etiology and age at onset are well known, but a history of febrile seizures is an unexpected predictive variable, of significant value only in those with idiopathic epilepsy. In this study, the duration and frequency of recurrence of febrile seizures are not noted, factors known to correlate with spontaneous seizures (Millichap, 1960). Intractability is defined as a terminal remission of less than one year and longest remission of less than 3 months during the last year of observation despite adequate treatment (optimal use of at least 2 AEDs, alone or in combination). In a group of children refractory for at least 2 years, $4 \%$ may enter remission during each year of follow-up (Huttenlocher and Hapke, 1990). The present paper emphasizes the changing course of epilepsy during childhood and underscores the need for a better definition of intractability when deciding on the timing of epilepsy surgery.

\title{
SPONTANEOUS REMISSION OF INTRACTABLE EPILEPSY FOLLOWING ACUTE VIRAL INFECTIONS
}

Questionnaires regarding the occurrence of seizure remission following infection were sent to pediatric neurologists in 73 university hospitals, children's hospitals, and epilepsy centers in Japan, and the data obtained in 21 cases from 11 institutions were analyzed at St Marianna University School of Medicine, Kawasaki, and other centers. Patients included were those whose frequent seizures had remitted for at least 1 month after viral infection without change in antiepileptic therapy. Age ranged from 6 months to 17 years. Sixteen ( $80 \%$ ) had West syndrome (13 symptomatic and 3 cryptogenic etiology), and the remainder included 2 cases of symptomatic generalized epilepsy, symptomatic partial epilepsy, continuous spike-wave and slow sleep (CSWS), and severe myoclonic epilepsy of infancy. Infections included 4 cases of exanthem subitum, 4 of rotavirus gastroenteritis, 3 of measles, 3 upper respiratory infections, 1 of mumps and cytomegalovirus, and 5 common cold. Seizures disappeared an average of 4.5 days (range, 1-14 days) after onset of infection. Follow-up was 34 months (range, 3 months to 4 years). Seizures recurred in 13 patients after a median of 7 months (range 1-30 months). Of 5 patients without seizure recurrence, 4 had West syndrome and 1 had CSWS. EEGs improved during remission periods in two-thirds of patients. AED serum levels showed no significant change during remission. Possible reasons for seizure remission included increased levels of antibodies after viral infection or suppression of immunological processes by anti-inflammatory cytokines. (Yamamoto $\mathrm{H}$, Yamano T, Niijima S et al. Spontaneous improvement of intractable epileptic seizures 
following acute viral infections. Brain Dev September 2004;26:377-379). (Respond: Dr H Yamamoto by E-mail: h3yama@marriana-u.ac.jp).

COMMENT. The authors cite 2 previous references to the remission of infantile spasms in West syndrome following viral infection, especially exanthem subitum (West, 1841; Hattori, 2001). Exanthem subitum and infection with human herpesvirus (HHV)-6 accounts for one-third of all first-time febrile seizures in children $<2$ years old in the US (Hall, Epstein, et al.1994). The virus that causes seizures may also have a protective effect in some patients. An elevated cytokine response independent of the severity of infection may be a factor in the mechanism of seizures (Kawada et al, 2003; Millichap JG, Millichap JJ, 2003), and suppression of immunological processes by anti-inflammatory cytokines (eg interleukin-10) may explain the seizure remission following viral infection in the above casereports. Further studies of the role of viral infections in the cause and remission of seizures are needed. (See Ped Neur Briefs April 2001, and Sept 2003, for further comment on cytokines and seizures).

\section{CARDIAC IRREGULARITIES AND TEMPORAL LOBE EPILEPSY}

Ictal and peri-ictal heart rate (HR) abnormalities in 20 children and adolescents with medically refractory symptomatic temporal lobe epilepsy (TLE) were investigated at the University Hospital Vienna, Austria. None had a known cardiac abnormality, and all were at rest in bed during EKG and EEG long-term monitoring. Of 72 TLSs analyzed, ictal tachycardia occurred in $71(98 \%)$, and ictal bradycardia was not observed. In pre-ictal stages, tachycardia occurred in 20 seizures and mild bradycardia in 3 . Tachycardia persisted $>60$ seconds after EEG seizure termination in 44 seizures (62\%). Rhythm and conduction abnormalities, including bradycardia, extreme HR oscillation, and premature ventricular beats, occurred in the postictal period during some complex partial TLSs. Early and high HR increase was associated with right mesial TLSs. The results confirm a right hemispheric lateralization of sympathetic cardiac regulation. (Mayer H, Benninger F, Urak L, et al. EKG abnormalities in children and adolescents with symptomatic temporal lobe epilepsy. Neurology July (2 of 2) 2004;63:324-328). (Reprints: Dr M Feucht, Universitatsklinik fur Neuropsychiatrie des Kindes- and Jugendalters, Wahringer Gurtel 18-20, 1090 Vienna, Austria).

COMMENT. Ictal tachycardia is the most frequent cardiac irregularity associated with complex partial seizures, occurring especially with epileptiform discharges lateralized to the right temporal lobe. It is suggested that EKG abnormalities and cardiac dysregulation during symptomatic temporal lobe epilepsy may contribute to the mechanism of sudden unexplained death.

The effect of hippocampal sclerosis on cardiovascular autonomic regulation was studied in 8 TLE patients with and 31 without hippocampal sclerosis (HS), using cardiovascular reflex tests and spectral analysis of 24-h ECG recordings, at University of Oulu, Finland. Compared to controls, a diminished heart rate variability (HRV) was observed in patients with, or without HS. Functional rather than structural changes are important in the altered cardiovascular regulation in patients with TLE. (Ansakorpi $\mathrm{H}$ et al. Epilepsia Aug 2004;45:933-939). 